\title{
Octahedral Engineering of Orbital Polarizations in Charge Transfer Oxides
}

\author{
Antonio Cammarata* and James M. Rondinelli ${ }^{\dagger}$ \\ Department of Materials Science $\mathcal{E}$ Engineering, Drexel University, Philadelphia, PA 19104, USA
}

(Dated: March 21, 2022)

\begin{abstract}
Negative charge transfer $A B \mathrm{O}_{3}$ oxides may undergo electronic metal-insulator transitions (MIT) concomitant with a dilation and contraction of nearly rigid octahedra. On both sides of the MIT are in-phase or out-of-phase (or both) rotations of adjacent octahedra that buckle the $B-\mathrm{O}-B$ bond angle away from $180^{\circ}$. Using density functional theory with the PBEsol $+U$ approach, we describe a novel octahedral engineering avenue to control the $B 3 d$ and $\mathrm{O} 2 p$ orbital polarization through enhancement of the $\mathrm{BO}_{6}$ rotation "sense" rather than solely through conventional changes to the $B-\mathrm{O}$ bond lengths, i.e. crystal field distortions. Using $\mathrm{CaFeO}_{3}$ as a prototypical material, we show the flavor of the octahedral rotation pattern when combined with strain-rotation coupling and thin film engineering strategies offers a promising avenue to fine tune orbital polarizations near electronic phase boundaries.

PACS numbers: 75.25.Dk, 61.50.-f, 71.20.-b
\end{abstract}

\section{INTRODUCION}

Transition metal oxide (TMO) perovskites are known to be strongly correlated materials, ${ }^{1}$ whose properties are controlled by a complex interplay between geometric and electronic degrees of freedom. These are determined by considering the relative magnitude between various energy scales and interactions: the energy difference of the transition metal $(M) d$ orbitals and the oxygen $p$ states, referred to as the charge transfer energy, and the strength of the on-site Hubbard $U$ interaction of the $d$ electrons. The charge transfer energy is more important in TMO where low-energy excitations are of $p-d$-type, whereas the Coulombic interaction, which localizes the electrons on the $M$-site, produces the insulating state in Mott-Hubbard systems. ${ }^{2}$ The properties of correlated electrons of TMO are controlled in part by the relative occupancy of the different transition-metal $d$ orbitals. ${ }^{3}$ The relative $d$ orbital occupancy is largely determined by the crystal field experienced by the transition-metal cation; this electrostatic field is the result of the $2 p$ electronic density of the coordinating oxygen ligands. The latter, in turn, is directed by the extended geometric arrangement of the nearest neighboring oxygen atoms.

Orbital occupancy can be tuned, for example, through chemical substitution, ${ }^{4,5}$ epitaxial strain, $, 6,7$ or by superlattice formation in thin films. ${ }^{8-10}$ Isovalent substitutions are important to charge transfer-type oxides, because rather than modifying the $M$-site electronic configuration, cations with different ionic sizes but the same formal valence renormalize the transfer interaction and the oneelectron bandwidths through changes in the crystal structure, i.e., interatomic bond angles and distances. The crucial distortion in $A B \mathrm{O}_{3}$ perovskites is the buckling of the $B-\mathrm{O}-B$ bond angles, because the effective $d$ electron transfer interaction between the neighboring transition metal sites is mediated by the angular overlap with the $\mathrm{O}$ $2 p$ states. ${ }^{11}$ When the $B-\mathrm{O}-B$ bond angle deviates from the ideal value of $180^{\circ}$, the transfer interaction weakens and the bandwidth narrows. Such distortions to the inter-octahedral bond angles are typical in $\mathrm{GdFeO}_{3}$-type perovskites, and therefore knowing how to control the amplitude of the bond angles distortions is critical to tailoring the charge transfer and $d$-orbital polarization. While it is well-established that greater octahedral rotations produce more buckled bond angles and narrower bandwidth, ${ }^{12}$ it is not well-understood how the rotation "sense" - be it in-phase or out-of-phase along a specific Cartesian direction - influences the differential charge occupancy on the $B$-site $d$ and O $2 p$ orbitals.

In this work, we use density functional calculations to show that controlling the subtle flavor of the octahedral rotation sense is as important as the overall amplitude of the rotations when engineering the electronic structure, vis-à-vis orbital polarization, of charge transfer oxides near electronic MIT. We explain this behavior using the definition of orbital polarization $\mathcal{P}$ of $m_{l 1}$ orbital relative to $m_{l 2}$ orbital,

$$
\mathcal{P}_{l_{1} m_{l 1}, l_{2} m_{l 2}}=\frac{n_{l_{1} m_{l 1}}-n_{l_{2} m_{l 2}}}{n_{l_{1} m_{l 1}}+n_{l_{2} m_{l 2}}},
$$

where $n_{l_{1} m_{l 1}}$ and $n_{l_{2} m_{l 2}}$ are the occupancies of $\left|l_{1} m_{l 1}\right\rangle$ and $\left|l_{2} m_{l 2}\right\rangle$ orbitals, with orbital quantum number $l_{i}$ and magnetic quantum number $m_{l i}$, respectively. ${ }^{8}$ With this definition, orbital polarization becomes an effective measure of the charge excess in the former orbital with respect to the latter. The metric then enables us to isolate the contribution of each rotation sense to the orbital polarization by judicious choice of structural distortions and subsequent calculation of $\mathcal{P}$.

We demonstrate this utility for the specific case of the Fe $3 d$ ( $e_{g}$-symmetry) and $\mathrm{O} 2 p$ orbitals in $\mathrm{CaFeO}_{3}$. Specifically, we show that out-of-phase octahedral rotations drive a transfer of electronic charge to the apical $2 p$ orbitals along the $\mathrm{Fe}-\mathrm{O}$ chains collinear with the axes of rotation, whereas the $\mathrm{Fe} e_{g}$ state are less sensitive. We then use tensile epitaxial strain as a practical handle to enhance the orbital polarization using the $\mathcal{P}$-contribution from the out-of-phase octahedral rotations to tune the ligand charge density - a crucial parameter involved in 
oxide-based MIT materials. Our results indicate that electronic-structure engineering requires both the amplitude and sense of the octahedral rotations be considered on equal footing when designing perovskite TMO for integration into next-generation electronics. ${ }^{13}$

\section{STRUCTURAL AND COMPUTATIONAL DETAILS}

To explore the rotation-orbital polarization interactions, we make use of the prototypical charge transfer oxide $\mathrm{CaFeO}_{3}$ (CFO), because it lacks Jahn-Teller distortions $^{14,15}$ and exhibits a sequence of structural phase transitions concomitant with a first-order MIT near room temperature (RT). ${ }^{16,17}$ Upon cooling below $290 \mathrm{~K}$, the metallic paramagnet becomes semiconducting (space group $\left.P 2_{1} / n\right)$, ${ }^{18}$ whereby the appearance of an octahedral breathing distortion (BD) together with the octahedral rotations, acts to open the electronic gap, ${ }^{19}$ as it has also been observed in rare-earth nickelates. ${ }^{20}$

Besides the octahedral breathing distortion, which consists of uniform elongation and contraction of $\mathrm{Fe}-\mathrm{O}$ bond lengths of adjacent octahedra, $\mathrm{CaFeO}_{3}$ adopts $^{21}$ the most common $a^{-} a^{-} c^{+}$Glazer tilt pattern, ${ }^{22}$ with buckled FeO-Fe bond angles (Fig. 1). Glazer notation allows us to distinguish the relative sense of the octahedral rotations about Cartesian axes passing through the Fe cation. The $+(-)$ superscript indicates in-phase (out-of-phase) rotations about each $x$-, $y$ - and $z$-axis. This rotation pattern consists of in-phase rotations about the $z$-axis $\left(a^{0} a^{0} c^{+}\right)$and is accompanied by two out-of-phase rotations, $a^{-} a^{0} c^{0}$ and $a^{0} a^{-} c^{0}$, of the same magnitude about the $x$ - and $y$-axes, respectively.

For a perovskite with a Bravais lattice that has orthogonal interaxial angles and the $a^{-} a^{-} c^{+}$pattern, the two important $\mathrm{Fe}-\mathrm{O}-\mathrm{Fe}$ bond angles are $\theta_{x y}$ and $\theta_{z}$ (Fig. 1). When only in-phase $a^{0} a^{0} c^{+}$or out-of-phase $a^{0} a^{0} c^{-}$rota-

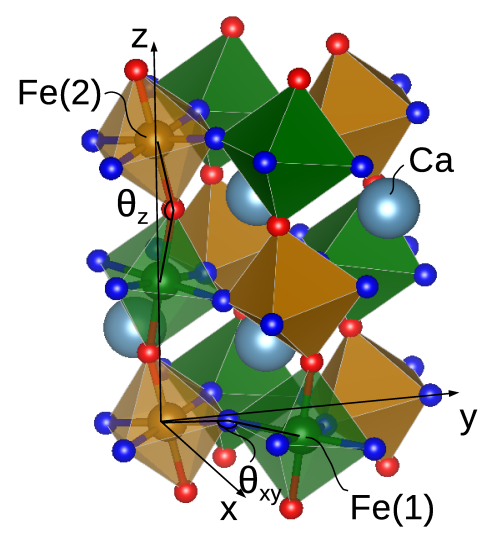

FIG. 1. Rotationally distorted $\mathrm{CaFeO}_{3}$ structure with the $8 d$ (equatorial) and $4 c$ (apical) oxygen Wyckoff positions indicated by blue and red spheres, respectively. Pseudo-cubic axes $(x, y$, and $z$ ) and $\mathrm{Fe}-\mathrm{O}-\mathrm{Fe}$ bond angles $\theta_{x y}$ and $\theta_{z}$ are also shown. tion patterns are present, the $\theta_{x y} \mathrm{Fe}-\mathrm{O}-\mathrm{Fe}$ angle measured within two subsequent $\mathrm{Fe}-\mathrm{O}$ layers orthogonal to the $z$ direction is the same. The phase, i.e., the "rotation sense," does not change the rotation magnitude across the layers. Naïvely, one would deduce that the electronic structure, and in turn the orbital polarizations, is insensitive to the sense of the rotations, since both rotations induce the same rotation magnitude about the $z$-axis - we investigate this supposition explicitly.

To evaluate how the rotation sense alters the electronic distribution, we carry out density functional calculations using the projector-augmented wave (PAW) formalism ${ }^{23}$ as implemented in the Vienna $A b$ initio Simulation Package (VASP) ${ }^{24}$ within the revised Perdew-Burke-Ernzerhof (PBE) generalized gradient approximation for densely packed solids $^{25}$ plus Hubbard $U \operatorname{method}^{26}(\mathrm{PBEsol}+U)$ based on the electronic and atomic structural analysis reported in our previous study. ${ }^{19}$ We chose the spherically averaged form of the rotationally invariant effective $U$ parameter of Dudarev et al. ${ }^{28}$ with a $U_{\text {eff }}=3.0 \mathrm{eV}$, hereafter $U$, on the $\mathrm{Fe} d$ orbitals. Note that no significant differences in the main features of the MIT occur for values up to $U$ values of $4.0 \mathrm{eV}$, as previously reported in literature, ${ }^{18}$ and we treat the double-counting term within the fully localized limit. ${ }^{27}$ We impose FM order on all Fe-sites then fully relax the spin density. We use a plane wave energy cutoff of $600 \mathrm{eV}$, which is then increased up to $850 \mathrm{eV}$ for calculations of the electronic densities of states (DOS). Ground state structures (DOS) are obtained by sampling the Brillouin zone with a minimum of a $7 \times 7 \times 7(9 \times 9 \times 7) k$-point mesh and the integrations performed with 20 meV Gaussian smearing (tetrahedron method). Atomic position relaxation are stopped when Hellmann-Feynman forces are minimized to a $0.5 \mathrm{meV}$ $\AA^{-1}$ tolerance.

\section{RESULTS AND DISCUSSION}

Our starting point is the analysis of the electronic DOS of cubic $\mathrm{CaFeO}_{3}$ without any octahedral rotations [Fig. 2(a)]. The features of interest are in the energy range -8 to $3 \mathrm{eV}$. The $\mathrm{O} 2 p$ states dominate the entire range, validating the charge transfer description. An energy gap appears at the Fermi level $\left(E_{F}\right)$ in the spin-down channel. The Fe $3 d$ orbitals contribute to the spin-down states with a main band centered near $-5 \mathrm{eV}$. In the spin-up channel, the Fe $3 d$ states are divided into two principal bands centered about -6.6 and $-2.2 \mathrm{eV}$, respectively, contributing to the metallicity with a broad band extending up to 2 eV above $E_{F}$.

We now examine the symmetry reduction consequences from the octahedral rotations and breathing distortions (BD) on the electronic structure of the cubic phase. The $\mathrm{BD}$ mode is responsible for splitting of the $1 a$ Fe site in the undistorted structure $(P m \overline{3} m)$ into two Wyckoff positions, $2 b$ and $2 c$, each centered in smaller and larger octahedra arranged as in a 3D checkerboard (Fig. 1). Both the in- 


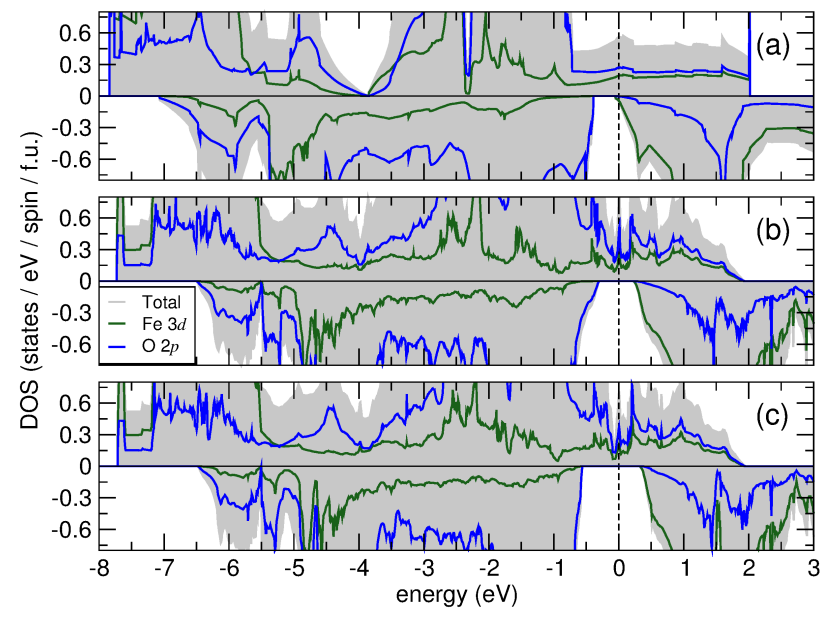

FIG. 2. Total and atom-resolved $\mathrm{CaFeO}_{3}$ density of states (DOS) for structures with (a) $\operatorname{Pm} \overline{3} m$, (b) $P 4 / n m c$ and (c) $I 4 / m$ symmetry. The structures in (b) and (c) are obtained by applying a breathing distortion together with $a^{0} a^{0} c^{+}$and $a^{0} a^{0} c^{-}$rotations to the cubic structure, respectively, at identical breathing amplitudes and $\theta_{x y}$ rotation angles but different rotation sense (cf. filled circles in Fig. 4). Differences at the Fermi level $(0.0 \mathrm{eV})$ induced by the two rotation senses are nearly indistinguishable.

phase $a^{0} a^{0} c^{+}$and the out-of phase $a^{0} a^{0} c^{-}$rotations split the $3 d$ oxygen site symmetries in the parent phase into two distinct sets; the consequence of these distortions is that the Fe $d$-orbitals experience an electrostatic interaction from the oxygen ligands which no longer has the complete octahedral cubic symmetry. Coupling the BD with inphase or out-of-phase rotations, results in structures with $P 4 / n m c$ or $I 4 / m$ tetragonal symmetry, respectively. This symmetry lowering changes the DOS with respect to the cubic phase mainly in energy windows far from $E_{F}$ : The spin-down bandwidth decreases, enhancing the band gap, and the spin-up states shift to higher energies in the conduction band (near $1 \mathrm{eV}$ ). Comparison of the DOS for the different rotation flavors [Fig. 2(b) and (c)], however, shows no appreciable differences near $E_{F}$; the low energy bands appear insensitive to the rotation sense.

\section{A. Orbital Polarization $-a^{0} a^{0} c^{+/-}$rotations}

We now track the change in the orbital polarization, $\mathcal{P}$, as we increase the breathing distortion together with the rotation amplitude of the oxygen displacement patterns associated with either the $a^{0} a^{0} c^{+}$or $a^{0} a^{0} c^{-}$rotations [Fig. 3(a) and Fig. 3(b)] about the $z$-direction. To compute $\mathcal{P}$, occupancies are calculated by integrating the angular momentum resolved DOS for particular $\left|l m_{l}\right\rangle$, obtained by projecting the Kohn-Sham wave functions onto the spherical harmonics, starting from the lower edge of the anti-bonding valence band states up to $E_{F}$.

The orbital polarization for the Fe $e_{g}$ states $(l=2)$, e.g., $\mathcal{P}_{d 2, d 0}$ which specifies the filling of the $d_{x^{2}-y^{2}}$ orbital

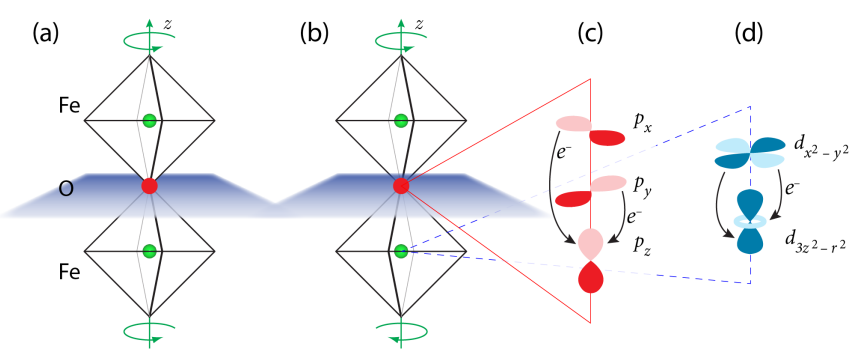

FIG. 3. (a) $a^{0} a^{0} c^{+}$and (b) $a^{0} a^{0} c^{-}$rotation patterns of two adjacent octahedra along the $z$-axis. (c) The out-of-phase rotations induce a transfer of charge from the equatorial plane (shaded), containing the $p_{x}$ and $p_{y}$ orbitals of the apical oxygen atom, to the $\mathrm{Fe}-\mathrm{O}$ apical bond about the $z$-axis, increasing the electron occupancy of the $p_{z}$ orbital lying along it. (d) On the Fe site, irrespective of the rotation sense, octahedral rotations induce charge transfer from the $d_{x^{2}-y^{2}}$ to the $d_{3 z^{2}-r^{2}}$ orbital, the latter directed along the apical $\mathrm{Fe}-\mathrm{O}$ bond.

$\left(m_{l}=2\right)$ relative to the $d_{3 z^{2}-r^{2}}$ orbital $\left(m_{l}=0\right)$ is $\mathcal{P}_{d 2, d 0}=\left(n_{x^{2}-y^{2}}-n_{3 z^{2}-r^{2}}\right) /\left(n_{x^{2}-y^{2}}+n_{3 z^{2}-r^{2}}\right)$, where $n_{x^{2}-y^{2}}$ and $n_{3 z^{2}-r^{2}}$ are the $d_{x^{2}-y^{2}}$ and $d_{3 z^{2}-r^{2}}$ orbital occupancies, respectively. We first analyze the orbital polarization of the apical [Fig. 4(a-d)] and equatorial oxygen atoms in $\mathrm{CaFeO}_{3}$ (see Fig. 1) for all possible $2 p$ orbital pairs with increasing amplitude of rotation and BD under a fixed volume constraint: $\mathcal{P}_{p x, p y}, \mathcal{P}_{p x, p z}$ and $\mathcal{P}_{p y, p z}\left(m_{l}=-1,0,1\right)$. Next, we evaluate $\mathcal{P}_{d 2, d 0}[\mathrm{Fe}(1)]$ and $\mathcal{P}_{d 2, d 0}[\mathrm{Fe}(2)]$, i.e., the orbital polarization of each unique Fe site.

The $\mathcal{P}_{p x, p y}$ polarization of the apical oxygen atoms evolves identically for both in-phase and out-of-phase rotations (data not shown); the small polarization value $(0.1 \%)$ also indicates that both $p_{x}$ and $p_{y}$ orbitals are equally populated, consistent with the tetragonal constraints imposed by the Bravais lattice. Over the structure-distortion space examined, ${ }^{29}$ we find the $\mathcal{P}_{p x, p z}$ and $\mathcal{P}_{p y, p z}$ polarization is positive with $p_{x}$ and $p_{y}$ preferentially occupied over the orbital directed along $z\left(p_{z}\right)$. Both polarizations are $\approx 10 \%$ and the width of variation is $4 \%$. These axial polarizations diminish for both rotation flavors if the BD increases. We understand this behavior as follows: The $p_{z}$ orbital pointing along $\mathrm{Fe}-\mathrm{O}$ bond axis increases its electron occupancy owing to a charge transfer from the equatorial plane, containing $p_{x}$ and $p_{y}$ orbitals, towards the $\mathrm{Fe}-\mathrm{O}-\mathrm{Fe}$ bond axis [Fig. 3(c)].

The orbital polarization involving $p_{z}$ is nearly constant with respect to increasing in-phase rotation and turns out to have a minimum at large out-of-phase angles and large breathing distortion values. At fixed BD amplitude, large out-of-phase angles direct the charge transfer towards the $\mathrm{Fe}-\mathrm{O}$ bond along the (rotation) $z$-axis

For the equatorial oxygen atoms, $\mathcal{P}_{p x, p y}$ is also small $(0.1 \%)$, indicating the orbitals are equally populated (data not shown). $\mathcal{P}_{p x, p z}$ and $\mathcal{P}_{p y, p z}$ have smaller values (1\%) compared to the apical oxygen atoms in all distortion ranges examined. For large, physically unreasonable distortions, the $p_{z}$ orbital not participating in the in-plane 


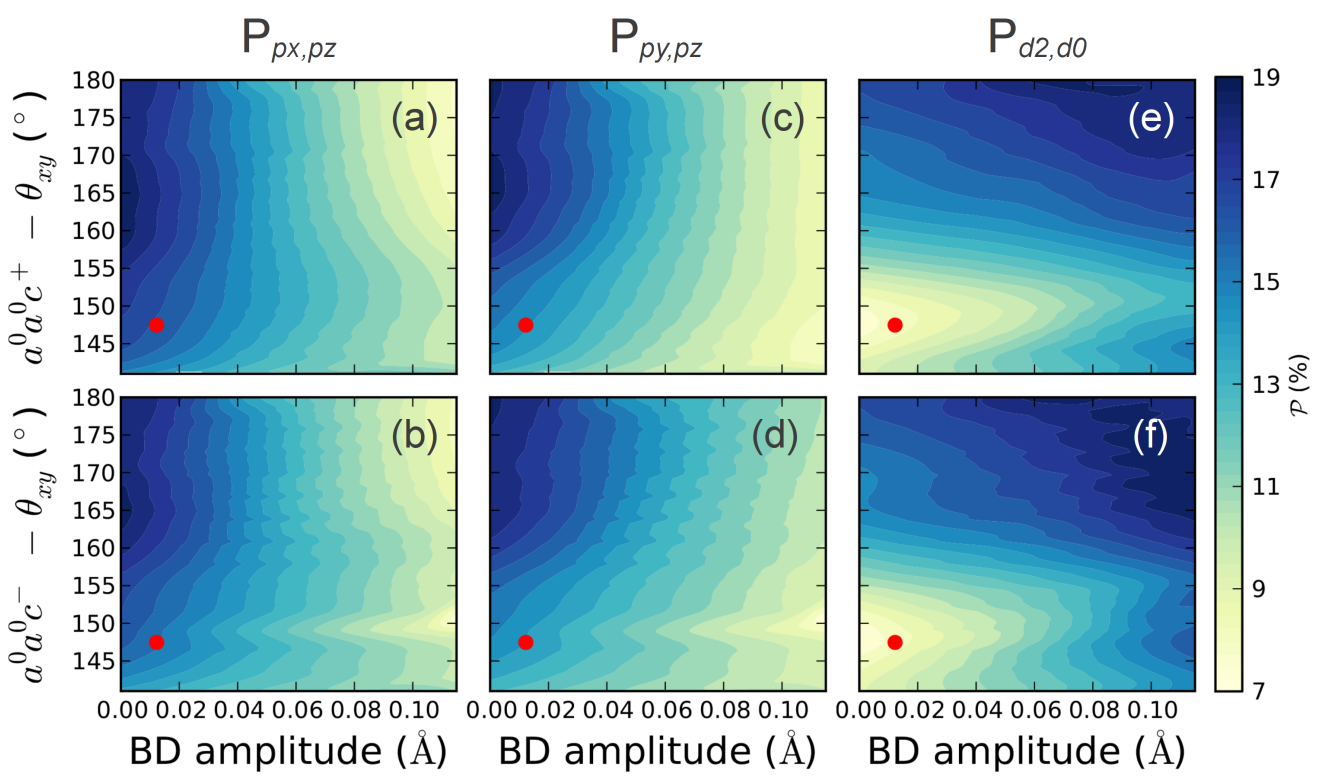

FIG. 4. Orbital polarizations for the apical oxygen atoms $\mathcal{P}_{p x, p z}$ (a) and (b), $\mathcal{P}_{p y, p z}$ (c) and (d), and Fe(1) site $\mathcal{P}_{d 2, d 0}(\mathrm{e})$ and (f) for breathing distortions (BD) coupled with in-phase (upper panels) and out-of-phase (lower panels) rotation patterns, respectively. $a^{0} a^{0} c^{-}$rotations induce charge transfer to the $p_{z}$ orbital aligned along the $\mathrm{Fe}-\mathrm{O}$ apical bond axis; Fe $e_{g}$ polarization is weakly affected by rotation sense. Filled (red) circles indicate the BD amplitude and the rotation angles for the structures whose DOS are given in Fig. 2(b) and (c).

Fe-O bond eventually becomes populated. Note that oxygen orbital polarizations involving $p_{z}$ are not affected by small breathing distortion values; however, coupling a large breathing amplitude with large rotation angles makes $\mathcal{P}$ maximum.

We now examine the Fe $3 d$-orbitals with $e_{g}$ symmetry. $\mathrm{Fe}(1)$ orbital polarization $\mathcal{P}_{d 2, d 0}[\mathrm{Fe}(1)]$ increases with breathing distortion [Fig. 4(e-f)], i.e., charges flow from the apical to equatorial bond axes. With decreasing amplitude of the rotation angle $\theta_{x y}$, the $d_{3 z^{2}-r^{2}}$ becomes preferentially occupied [Fig. 3(d)]. For $\mathrm{Fe}(2)$ the $\mathcal{P}_{d 2, d 0}[\mathrm{Fe}(2)]$ decreases as the charge flow reverses from the equatorial to apical bond. Both polarizations are minimal for small rotation angle values (further from cubic symmetry), yet the differential occupation appears to be weakly dependent on the rotation sense. The out-of-phase rotations, however, influence the $e_{g}$ orbitals more than the in-phase ones.

To summarize to here, our analysis reveals that the apical oxygen atoms lying along the rotation axis are mainly sensitive to the octahedral rotation sense, with out-of-phase rotations favoring charge flow to the $\mathrm{Fe}-\mathrm{O}$ apical bond. The orbital occupancy of the equatorial oxygen atoms is weakly affected by the rotation pattern sense. ${ }^{30}$ Moreover, the iron sites are largely sensitive to the breathing distortion, whereby the more expanded an octahedron becomes, the greater is the charge transfer to the apical bond axes. This effect increases the orbital polarization of the corresponding Fe cations; it can also be more readily achieved with the $a^{0} a^{0} c^{-}$rather than the $a^{0} a^{0} c^{+}$rotation pattern. These results suggest that sense of the octahedral rotation mainly affects the oxygen atoms lying along the rotation axis that "screen" the metal cations, which are unable to "see" the rotation sense effects.

\section{B. Orbital Polarization with Multiple Rotations}

In the experimental $\mathrm{CaFeO}_{3}$ structure, the $\mathrm{BD}$ is present together with the $a^{-} a^{-} c^{+}$rotation pattern, where both rotation senses are present and out-of-phase rotations are found about two Cartesian directions. We now analyze the orbital polarization in $\mathrm{CFO}$ structures where the $\mathrm{BD}$ is coupled with the $a^{-} a^{-} c^{0}$ rotation to evaluate the conclusions about the different rotation sense discussed above in the presence of multiple rotation axes.

We find polarizations $\mathcal{P}_{p x, p y}, \mathcal{P}_{p x, p z}$ and $\mathcal{P}_{p y, p z}$ of the apical oxygen atoms increase when the rotation angle $\theta_{z}$ approaches $180^{\circ}$ (data not shown). ${ }^{31}$ As before, the outof-phase rotations will transfer charge from the orbitals lying in the plane normal to the rotation axes to the orbital lying along the axes. In this case, the rotations in the $x y$-plane induce a flow of charge from $p_{z}$ to both the $p_{x}$ and $p_{y}$ orbitals, i.e. from the $\mathrm{Fe}-\mathrm{O}$ apical bond into the plane orthogonal to it and containing the apical oxygen atom and its $p_{x}$ and $p_{y}$ orbitals. On the other hand, the equatorial oxygen atoms experience charge flow to their corresponding $\mathrm{Fe}-\mathrm{O}$ bonds. Concerning the two Fe sites, decreasing the $\theta_{z}$ rotation angle moves charge from the apical $\left(d_{3 z^{2}-r^{2}}\right.$ orbital $)$ to the equatorial $\left(d_{x^{2}-y^{2}}\right)$ axis. Here, the $\mathrm{BD}$ has the role of enhancing the charge transfer induced by the $a^{-} a^{-} c^{+}$rotation pattern. This last result, together with the analysis of the single in- 
phase and out-of-phase case discussed above, reveals that the out-of-phase rotation pattern favors a flow of charge towards its corresponding rotation axis. The rotation "sense" becomes an important structural feature over the orbital population.

In $\mathrm{CaFeO}_{3}$, the $\mathrm{Fe}-\mathrm{O}$ equatorial bonds are known to play a fundamental role in the metal-insulator transition: ${ }^{19}$ a change in the equatorial bond-lengths together with the octahedral rotations affects the $d_{x^{2}-y^{2}} /\left(p_{x}, p_{y}\right)$ orbital hybridization responsible for the formation of a highly covalent electronic configuration, gapping the system. An alternative route for tuning the band gap emerges from our results. If the $\mathrm{Fe}-\mathrm{O}$ bond lengths are not altered, then the $a^{-} a^{-} c^{0}$ out-of-phase rotation pattern controls the amount of charge filling the equatorial orbitals, hence the degree of hybridization, which determines the stability of the insulating phase.

\section{Strain-rotation and Orbital Polarization Coupling}

Strain can enhance the octahedral rotations in perovskites ${ }^{32}$ which in turn alter the band structure. ${ }^{33-36}$ Tensile bi-axial strain promotes the $a^{-} a^{-} c^{+}$rotation pattern in $c$-oriented orthorhombic perovskites thin films. ${ }^{37}$ To explore the effect of strain on rotation-induced $\mathcal{P}$, we compute the equilibrium structure of $\mathrm{CaFeO}_{3}$ under biaxial tensile strain, relaxing ionic positions and the $c$-axis length.

First, we evaluate the effect of strain on the atomic structure. We find that $\theta_{z}$ deviates more from $180^{\circ}$ with increasing tensile strain [Fig. 5(a)]. Consistent with our previous observation, when the $\mathrm{BD}$ and $a^{-} a^{-} c^{0}$ rotations

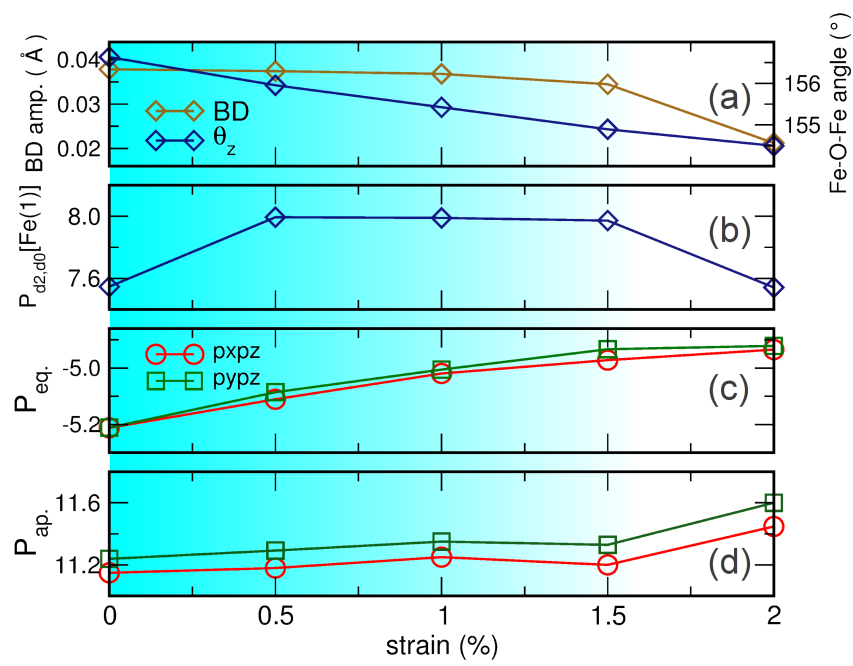

FIG. 5. BD amplitudes and $\theta_{z}$ angles (a), orbital polarization (\%) of (b) $\mathrm{Fe}(1)$ site, (c) equatorial (eq.) and (d) apical (ap.) oxygen atoms for relaxed $\mathrm{CaFeO}_{3}$ structures under biaxial strain. The shaded area indicates strain values for insulating structures; the MIT occurs between 1-1.5\% strain. are active, the orbital polarization is enhanced along the $\mathrm{Fe}-\mathrm{O}$ equatorial bonds with greater tensile strain. Importantly, we note that within the insulating phase as strain is increased, the BD largely remains constant while the octahedral rotations are enhanced to accommodate the strain energy [Fig. 5(a)]. The result of the decreased $\theta_{z}$ rotation angle is further charge localization in the $d_{x^{2}-y^{2}}$ [Fig. 5(b)] and $p_{x}$ and $p_{y}$ orbitals of equatorial oxygen atoms [Fig. 5(c)], hence about the equatorial $\mathrm{Fe}-\mathrm{O}$ bonds supporting the insulating state and leaving unaltered the polarization of apical oxygen atoms [Fig. 5(d)].

Our results show that the $\mathrm{O} 2 p$ and $\mathrm{Fe} e_{g}$ orbital polarizations are highly sensitive to the strain state and are mediated largely by the change in the out-of-phase octahedral rotations $\left(\theta_{z}\right)$. The tensile strain, enhances the $a^{-} a^{-} c^{0}$ rotation pattern, induces a charge transfer into those orbitals responsible for the gap (i.e. $d_{x^{2}-y^{2}}$, and $p_{x} / p_{y}$ of equatorial oxygen atoms): beyond a critical value, the charge becomes delocalized and the insulating gap closes to form a metallic state. We observe that the insulating phase is lost for strain values greater than $1 \%$, which is in contrast to that seen in rare-earth nickelates, ${ }^{20}$ suggesting the rotation-orbital polarization interaction and the BD may be either complementary or antagonistic to the MIT.

The out-of-phase rotations are also responsible for the broadening of the $p_{x}$ and $p_{y}$ orbitals about $E_{F}$, which we conjecture would reduce the sharpness of the first-order phase transition. Note that the critical transition temperature for the MIT is also believed to be correlated with the size of the BD, our results therefore suggest that strainengineering of the MIT temperature could be challenging in ferrates. On the other hand, the structural transition temperature can be kept fixed with increasing strain while the electronic transition can still be tuned by means of the octahedral rotation-induced orbital polarizations.

\section{CONCLUSIONS}

In summary, we have shown how the sense of the octahedral rotation pattern and not only the magnitude of the rotation distortion is important in engineering the low energy electronic band structure. We find that while the in-phase rotations weakly affect the local electronic distribution, the out-of-phase rotation pattern directs a flow of charge towards its corresponding rotation axis. The size of the rotation-sense orbital polarization we compute should be experimentally detectable with orbital reflectometry techniques, ${ }^{38}$ across the orthorhombic $\left(a^{-} a^{-} c^{+}\right)$ to monoclinic $\left(a^{-} b^{-} c^{+}\right)$phase transition of $\mathrm{CaFeO}_{3}$ near room temperature. Here, the out-of-phase rotation pattern is no longer uniform along [110]. For charge transfer perovskite oxides, control over the phase of the rotations via heteroepitaxial strain, substrate proximity effects, or superlattice formation provides a promising alternative route to tailor the local orbital polarizations, hence electronic transitions, in correlated materials near electronic 
phase boundaries. Due to the complexity of these interactions driven by cation substitution, we hope this work motivates further experimental studies to evaluate the mechanisms proposed here, and the degree to which they compete or cooperate across a MIT.

\section{ACKNOWLEDGMENTS}

A.C. and J.M.R. were supported by ONR under grant no. N00014-11-1-0664. This work used the Extreme Science and Engineering Discovery Environment (XSEDE), which is supported by NSF (OCI-1053575). The use of VESTA software is also acknowledged. ${ }^{39}$
* acammarata@coe.drexel.edu

† jrondinelli@coe.drexel.edu

1 E. Dagotto, Science 309, 257 (2005).

2 M. Imada, A. Fujimori, and Y. Tokura, Rev. Mod. Phys. 70, 1039 (1998).

3 Y. Tokura and N. Nagaosa, Science 288, 462 (2000).

${ }^{4}$ M. B. Salamon and M. Jaime, Rev. Mod. Phys. 73, 583 (2001).

5 P. Werner and A. J. Millis, Phys. Rev. Lett. 99, 126405 (2007).

6 J. W. Freeland, J. Liu, M. Kareev, B. Gray, J. W. Kim, P. Ryan, R. Pentcheva, and J. Chakhalian, Europhys. Lett. 96, 57004 (2011).

7 E. J. Moon, J. M. Rondinelli, N. Prasai, B. A. Gray, M. Kareev, J. Chakhalian, and J. L. Cohn, Phys. Rev. B 85, 121106 (2012).

${ }^{8}$ M. J. Han, C. A. Marianetti, and A. J. Millis, Phys. Rev. B 82, 134408 (2010).

9 J. Liu, S. Okamoto, M. van Veenendaal, M. Kareev, B. Gray, P. Ryan, J. W. Freeland, and J. Chakhalian, Phys. Rev. B 83, 161102 (2011).

10 P. Hansmann, A. Toschi, X. Yang, O. K. Andersen, and K. Held, Phys. Rev. B 82, 235123 (2010).

11 J. K. Burdett, Inorg. Chem. 14, 931 (1975).

12 G.-Q. Liu, Phys. Rev. B 84, 235136 (2011).

13 S. D. Ha and S. Ramanathan, J. App. Phys. 110, 071101 (2011).

14 Y. Tomioka, T. Okuda, Y. Okimoto, A. Asamitsu, H. Kuwahara, and Y. Tokura, J. All. Comp. 326, 27 (2001).

15 M. H. Whangbo, H. J. Koo, A. Villesuzanne, and M. Pouchard, Inorg. Chem. 41, 1920 (2002).

16 S. K. Park, T. Ishikawa, Y. Tokura, J. Q. Li, and Y. Matsui, Phys. Rev. B 60, 10788 (1999).

17 M. Mostovoy, J. Phys.: Condens. Matter 17, S753 (2005); Phys. Rev. Letters 94, 137205 (2005).

18 T. Saha-Dasgupta, Z. S. Popović, and S. Satpathy, Phys. Rev. B 72, 045143 (2005).

19 A. Cammarata and J. M. Rondinelli, Phys. Rev. B 86, 195144 (2012).

20 S. Lee, R. Chen, and L. Balents, Phys. Rev. B 84, 165119 (2011); J. A. Alonso, J. L. García-Muñoz, M. T. FernándezDíaz, M. A. G. Aranda, M. J. Martínez-Lope, and M. T. Casais, Phys. Rev. Lett. 82, 3871 (1999); H. Park, A. J. Millis, and C. A. Marianetti, Phys. Rev. Lett. 109, 156402 (2012); S. Prosandeev, L. Bellaiche, and J. Íñiguez, Phys. Rev. B 85, 214431 (2012).

21 Strictly, the low-temperature insulating monoclinic phase adopts the $a^{-} b^{-} c^{+}$, and thus the angle of rotations about each axis are unique, but these deviations in the bond angles are small.

22 A. M. Glazer, Acta Cryst. B 28, 3384 (1972).

23 P. E. Blöchl, Phys. Rev. B 50, 17953 (1994).
${ }^{24}$ G. Kresse and J. Furthmüller, Comput. Mat. Sci. 6, 15 (1996); G. Kresse and D. Joubert, Phys. Rev. B 59, 1758 (1999).

25 J. P. Perdew, K. Burke, and M. Ernzerhof, Phys. Rev. Lett. 77, 3865 (1996); J. P. Perdew, A. Ruzsinszky, G. I. Csonka, O. A. Vydrov, G. E. Scuseria, L. A. Constantin, X. Zhou, and K. Burke, Phys. Rev. Lett. 100, 136406 (2008).

${ }^{26}$ V. I. Anisimov, F. Aryasetiawan, and A. I. Lichtenstein, J. Phys.: Condens. Matter 9, 767 (1997).

27 E. R. Ylvisaker, W. E. Pickett, and K. Koepernik, Phys. Rev. B 79, 035103 (2009).

28 S. L. Dudarev, G. A. Botton, S. Y. Savrasov, C. J. Humphreys, and A. P. Sutton, Phys. Rev. B 57, 1505 (1998).

29 Fig. 4 is generated from 345 structures and performing self-consistent total energy calculations; linear interpolation of the computed orbital polarizations yields the full rotation-BD orbital polarization surfaces. We apply a twodimensional moving-average filter to smooth the data for clarity.

30 When only the breathing distortion is present, the compound becomes insulating at high $\mathrm{BD}$ amplitudes $(\geq 0.13$ $\AA)$. If the $\mathrm{BD}$ is absent, rotations alone do not induce a metal-insulator transition. ${ }^{19}$ At fixed BD, however, there are some dependencies of the electronic band gap on the rotation sense.

31 Identical conclusions are obtained from considering $\theta_{x y}$ instead of $\theta_{z}$, i.e., when the breathing distortion is coupled with $a^{-} a^{-} c^{0}$ rotations, $\theta_{x y}$ is an increasing monotonic function of $\theta_{z}$.

32 J. M. Rondinelli, S. J. May, and J. W. Freeland, MRS Bulletin 37, 261 (2012).

${ }^{33}$ R. F. Berger, C. J. Fennie, and J. B. Neaton, Phys. Rev. Lett. 107, 146804 (2011).

34 J. M. Rondinelli and S. Coh, Phys. Rev. Lett. 106, 235502 (2011).

35 B. Dupé, S. Prosandeev, G. Geneste, B. Dkhil, and L. Bellaiche, Phys. Rev. Lett. 106, 237601 (2011).

36 S. Lv, H. Li, Z. Wang, L. Han, Y. Liu, X. Liu, , and J. Meng, Appl.Phys. Lett. 99, 202110 (2011).

37 J. M. Rondinelli and N. A. Spaldin, Advanced Materials 23, 3363 (2011).

38 A. Benckiser, M. Haverkort, S. Brck, E. Goering, S. Macke, A. Fra, X. Yang, O. Andersen, G. Cristiani, H.-U. Habermeier, A. V. Boris, I. Zegkinoglou, P. Wochner, H.-J. Kim, V. Hinkov, and B. Keimer, Nat. Mat. 10, 189 (2011).

39 K. Momma and F. Izumi, J. Appl. Cryst. 41, 653 (2008). 